\title{
Nano-scale mechanism of crack nucleation/propagation and lithium penetration in solid electrolyte
}

\section{Megan Diaz}

University of Central Florida

Qingsong Tu

Rochester Institute of Technology

Akihiro Kushima ( $\nabla$ Kushima@ucf.edu)

University of Central Florida https://orcid.org/0000-0001-5166-4198

\section{Article}

Keywords:

Posted Date: February 18th, 2022

DOI: https://doi.org/10.21203/rs.3.rs-1346996/v1

License: (c) (i) This work is licensed under a Creative Commons Attribution 4.0 International License.

Read Full License 


\section{Abstract}

This work presents a direct observation and quantification of the fundamental mechanism of lithium penetration in Li6PS5Cl (LPS) solid electrolyte. Lithium plating in a nano-sized defect on the LPS surface led to the nucleation and propagation of a crack without external force. It also revealed a reduction in the mechanical strength of LPS when altering the electrochemical charge/discharge bias condition. A first principles atomistic simulation was performed to confirm that the disorder in the crystal structure of the LPS, both in lithium deficient and excess states, contributes to the reduced mechanical strength, and a decrease in the modulus is observed when lithium concentration is decreased from the stoichiometric amount. The results of this study suggest the importance of minimizing defects at the surface and grain boundaries to improve the stability of the solid-state electrolyte (SSE). Interfaces and boundaries can be bottlenecks for lithium diffusion, creating the concentration gradient. This can reduce the mechanical stabilities of the SSE, accelerating lithium penetration and degradation in all-solid-state lithium batteries. The insights obtained in this study provide useful information towards understanding the mechanism and designing the materials/structures to solve this issue.

\section{Introduction}

Although lithium ion batteries (LIBs) are dominating the current energy storage market, ${ }^{1-3}$ their performance is nearing the theoretical limits. ${ }^{4}$ Therefore, there is an urgent need for the development of next generation energy storage technologies to meet the requirements for highly demanding applications such as electric vehicles (EVs). Use of a lithium metal anode is key because of its high theoretical capacity of $3800 \mathrm{mAh} / \mathrm{g}$ and low electrochemical potential of $-3.04 \mathrm{~V}$ with respect to the standard hydrogen electrode. However, increased energy densities raise safety concerns from unexpected failures $^{5-9}$, and the use of a lithium metal anode increases the risk of the nucleation and growth of dendrites leading to a short circuit, fire, or even explosion. ${ }^{10,11} \mathrm{Here}$, all-solid-state lithium batteries (ASSLBs) offer a solution, with their promises of increased energy density and improved safety. The use of an solid-state electrolyte (SSE) with a much higher modulus than lithium metal is expected to prevent the penetration of the dendrites and allow for the use of a high capacity lithium metal anode without safety issues. ${ }^{12,13}$ In order to guarantee these promises, a few setbacks must still be overcome before solid state technology is used commercially in EVs and portable electronics. Two important issues include 1) low $\mathrm{Li}^{+}$conductivity in the solid electrolyte, ${ }^{14-17}$ which reduces the rate performance compared to the liquid-electrolyte-containing LIB counterpart, and 2) lithium dendrite penetration that ultimately causes failure of the battery due to shorting. ${ }^{18-22}$

The $\mathrm{Li}_{2} \mathrm{~S}-\mathrm{P}_{2} \mathrm{~S}_{5}$ based sulfide (LPS) is one of the promising solid state electrode materials for ASSLBs. It exhibits a high lithium-ion conductivity $\left(1.4 \times 10^{-5}\right.$ to $\left.1.33 \times 10^{-3} \mathrm{~S} \mathrm{~cm}^{-1}\right)$ at room temperature, ${ }^{23-26}$ a low electron conductivity $\left(3.40 \times 10^{-9} \mathrm{~S} \mathrm{~cm}^{-1}\right),{ }^{27,28}$ and a large electrochemical stability window of 0 to $7 \mathrm{~V}$ against lithium. ${ }^{25}$ However, LPS is no exception when it comes to the problem of lithium dendrites. ${ }^{27}$ Compressing the SSE against the electrodes is necessary for good contact in ASSLBs, but that 
compression force may also be leading to crack growth as lithium is forced into the pores of LPS. ${ }^{29}$ Crack growth is especially probable at defect areas in the LPS SSE and where lithium plating and stripping occur frequently. ${ }^{27}$ LPS has been shown to have a low fracture toughness and be quite brittle, ${ }^{30}$ making lithium plating in surface defects a major cause for concern. Moreover, cracks in SSEs have been shown to decrease ionic conduction, ${ }^{31}$ degrading one of the main benefits of LPS. Therefore, it is important to better understand the role of lithium in nucleation and growth of cracks in LPS SSEs.

The origin of lithium deposition and growth through the bulk LPS and the necessary conditions to avoid it are still disputed. ${ }^{32,33}$ However it has been theorized that lithium can accumulate in the SSE, ${ }^{27,34}$ and if near a large-enough crack, ${ }^{35}$ the force due to enough accumulation can pry the crack open, propagating crack growth ${ }^{36,37}$. Cracks formed via lithium plating encourage more lithium plating in that particular area, which in turn increases local electronic conductivity, lowering the energy barrier and promoting more dendrite growth and propagation. ${ }^{32}$ As discussed later in this article, the threshold force necessary to initiate crack growth is much lower than the maximum force of dendrites growing through the LPS SSE, revealing just how easy it is for dendrites to form in a defect area of the SSE and cascade into forming a rapid-dendrite growth area.

In situ transmission electron microscopy (TEM) is an invaluable technique to observe and analyze dynamic processes in nano scales. It has been used to study the lithium dendrite growth process. ${ }^{38-40}$ For example, lithium penetration through an $\mathrm{Li}_{6.4} \mathrm{La}_{3} \mathrm{Zr}_{1.4} \mathrm{Ta}_{0.6} \mathrm{O}_{12}$ (LLZTO) SSE was revealed, ${ }^{38}$ and a direct measurement of the growth force was conducted. ${ }^{39,40}$ However, the quantitative details on the electrochemomechanics during the penetration process is still missing. In this work, we focused on an individual defect on the LPS SSE surface, studied the dendrite penetration process and correlated it with the mechanical property of the SSE. We employed an in situ TEM technique developed to observe the lithium dendrite growth and quantify the force during the process in real time. ${ }^{39}$ The results provided direct evidence of lithium penetrating a SSE involving crack nucleation and growth initiating from a nanosized defect, which can lead to shorting and a failure of ASSLBs. Quantitative mechanical property analysis using in situ TEM, together with first principles simulation, revealed important insights on the electrochemomechanics at the Li/SSE interface in ASSLBs.

\section{Methods}

In situ transmission electron microscopy - We modified a prior in situ TEM setup used to analyze electrochemomechanincal behavior of Li dendrites. ${ }^{39}$ The experiment was conducted in an FEI Tecnai F30 TEM equipped with a Schottky field emission source at an operating voltage of $300 \mathrm{kV}$. The TEM sample holder used was a Hummingbird 3D-Nanomanipulation Holder equipped with a piezomanipulator and biasing capability. In an argon-filled glovebox, solid Li metal was scraped with a razor blade to remove its oxidized surface layer and expose a fresh surface. The Li metal was then scratched with a W rod, and LPS SSE powder (Ampcera Argyrodite $\mathrm{Li}_{6} \mathrm{PS}_{5} \mathrm{Cl}$ Sulfide Solid Electrolyte Powder, particle size $<10 \mu \mathrm{m}$ ) was placed on top of the small piece of Li metal that was attached on the tip. Since this 
process was completed in the glove box, it reduces the chance of a small lithium oxide layer forming between the lithium metal and SSE upon a short air exposure during the insertion of the holder into the TEM. The W rod with Li and LPS particles was mounted on the piezo-manipulator side of the holder. A conductive atomic force microscopy (AFM) cantilever (NANOSENSORS PPP-CONTSCPt) was affixed to a gold rod using conductive epoxy and mounted on the opposite end of the holder. A schematic illustration of the in situ TEM setup is shown in Fig. 1A. The holder was then sealed and transferred into the TEM to minimize the atmospheric exposure of the Li metal and LPS SSE ( $<2 \mathrm{~s}$ during insertion of the holder). Once inside the TEM, the W rod was approached to the cantilever tip until contact between the cantilever tip and LPS SSE was achieved at a target location on the LPS. A negative bias was applied to encourage lithium dendrite growth via lithium plating between the cantilever and LPS (Fig. 1B,C). Image analysis was performed to measure the displacement of the cantilever tip during lithium deposition to calculate the force imposed on the Li and SSE (Fig. 1D).

Ab initio simulation - Mechanical properties of the LPS were calculated using the Vienna Ab-initio Simulation Package (VASP). ${ }^{41,42}$ The initial simulation slab model consisted of $24 \mathrm{Li}, 4 \mathrm{P}, 20 \mathrm{~S}$ and $4 \mathrm{Cl}$ atoms. Various simulations were performed at differing amounts of $\mathrm{Li}$ atoms, with up to $20 \mathrm{Li}$ atoms removed (4 $\mathrm{Li}$ atoms total) to $5 \mathrm{Li}$ atoms added ( $29 \mathrm{Li}$ atoms total). The $\mathrm{Li}$ atoms were randomly removed or added and structural optimization was performed by the conjugate gradient method. Then, the simulation cell was stretched in the [100] direction followed by the relaxation of the atomic position and cell vectors while keeping the size constant in [100]. A plane-wave energy cut-off of $520 \mathrm{eV}$ and a generalized gradient approximation (GGA) parameterized by Perdew, Burke and Ernzerhof ${ }^{43}$ were used for the exchange-correlation functional. The ionic core was represented with a projector augmented wave (PAW) potential, ${ }^{44,45}$ and a $2 \times 2 \times 2$ Monkhorst-Pack ${ }^{46}$ k-point mesh was used.

\section{Results And Discussion}

Figure 1E-G shows the snapshots of the Li deposition in a defect causing the nucleation/growth of a crack (See also Supplementary Movie S1). First, a piezo manipulator from the in situ TEM holder was controlled to insert a cantilver tip in a surface defect on LPS to grow Li. The LPS SSE was approached to the cantilever, until the cantilever was aligned with and achieved contact inside the crack, as seen in Fig. $1 \mathrm{E}$. A negative potential $(-2 \mathrm{~V})$ on the cantilever with respect to the Li metal was then applied, which caused lithium plating inside the crack and initial crack opening. Over time, continued lithium plating increased the crack length and width. A snapshot after $118 \mathrm{~s}$ of biasing is shown in Fig. 1F. A small piece of lithium grew inside the defect (dashed line) pushing the crack open. As a result, nucleation of a sharp crack was observed at the defect tip shown by the arrowhead. As the deposition of lithium continued, the growing lithium further pushed the crack open and penetrated the LPS. This process created a new crack at the tip as shown in Fig. 1G. Here, there was no displacement of the cantilever tip indicating that the compressive stress at the interface between the lithium and the crack walls did not exceed the threshold value to cause transition in the growth direction. ${ }^{39}$ 
This crack nucleation/growth mechanism during lithium deposition is schematically explained in Fig. $1 \mathrm{H}$. Due to the present geometry, shown in the enlarged force vectors schematic, lithium pushed on the LPS SSE crack walls, which caused lithium growth primarily in the direction perpendicular to the cantilever. The lack of cantilever displacement was attributed to the lithium growth direction, which resulted in the inability to measure the forces imposed upon the LPS SSE. However, this in situ TEM observation revealed an important lithium penetrating mechanism and demonstrated that lithium plating into existing surface defects has the power to grow cracks deeper into the LPS SSE. These cracks propagate ahead of the penetrating lithium as shown by the empty space in the $118 \mathrm{~s}$ image in Fig. $1 \mathrm{~F}$, which agrees with an in-situ X-ray computed tomography and X-ray diffraction reported by Ning. ${ }^{47}$ Similar lithium plating experiments were performed selectively at flat defect-free areas on the SSE surface and confirmed no damage on the SSE was visible during the plating (See Supporting Information for details). This emphasizes the importance of pre-existing defects (or those formed during the multiple battery cycles) for lithium penetration through SSE.

The development of the stress at the defect was estimated using a simple fracture equation, assuming the lateral stress $(\sigma)$ is responsible for the crack (with length $a$ ) to open under fracture Mode I. Thus, the fracture equation $\sigma_{c}=K_{I C} / \sqrt{\pi a}$ should be satisfied. $\sigma_{c}$ is the minimal stress required to open a crack with length $a$, and $K_{I C}$ is the fracture toughness (for LPS, $K_{I C} \approx 0.23 \mathrm{MPa} \cdot{\sqrt{m^{30}}}^{\text {) }}$ ). Therefore, the stress required to open the crack in Fig. $1 \mathrm{~F}(a=146 \mathrm{~nm})$ and $1 \mathrm{G}(a=231 \mathrm{~nm})$ can be estimated to be $340 \mathrm{MPa}$ and $270 \mathrm{MPa}$, respectively. These values are significantly larger than the yield stress of Li metal. The extremely high aspect ratio of the nano-sized crack allowed continuous lithium addition at the tip without plastic flow and caused a stress build-up beyond the limit. ${ }^{37}$ Notably, the above evaluated stress values are the upper bound of the internal stress at the defect due to the existence of pores in LPS (with porosity $5 \sim 10 \%) .48$

To determine the forces involved during lithium plating-initiated crack growth, the AFM cantilever was approached towards a crack on the LPS surface at a tilted angle as shown in Fig. 2A. After the cantilever tip contacted the crack wall, a bias potential of $-7 \mathrm{~V}$ was applied on the cantilever side to initiate lithium plating. Under the bias, a lithium dendrite grew between the cantilever and the LPS inside the surface crack (see Supplementary Movie S2). The growth of the crack in the height and width direction, along with the force imposed on the crack wall, was analyzed and is discussed in depth in the following sections. In this setup, lithium was plated on one sidewall of the crack against the cantilever to push the crack open. Therefore, the force required to open and propagate a crack in LPS can be evaluated. The plated lithium remained between the cantilever and the LPS SSE, revealing that lithium can withstand the forces imposed throughout it during the crack growth.

Figure $2 \mathrm{~B}$ shows the change in the crack geometry and the force during the initial crack propagation, which consisted of two stages. The snapshots of the lithium plating and the crack propagation process are shown in Fig. 2C-E. The width $w$ and the length I are measured using the features of the crack as 
shown in Fig. $2 \mathrm{C}$. In the first stage, lithium started plating upon biasing. The force experienced by the crack increased due to the lithium plating between the cantilever and the crack wall, which was measured by the displacement of the cantilever tip (Fig. 2D). In this initial lithium plating (Stage I), the crack geometry remained constant, indicating the threshold stress for the initiation of crack growth had not been reached. When the crack threshold stress was reached, an increase in force caused both the crack length and width to increase (Stage II). This threshold force initiating crack growth was measured to be $34 \mathrm{nN}$. The exact stress experienced by the dendrite, and therefore the LPS, is difficult to precisely determine, since it was not possible to perform a rotation of the sample during the experiment that would allow for a view of the exact dimensions of the plated lithium. However, assuming circular cross sections, stress on the cantilever tip and the LPS can be estimated to be $0.3 \mathrm{Mpa}$ and $0.04 \mathrm{MPa}$, respectively. These values were smaller than the maximum stress estimated for the lithium plating on the flat defectfree LPS surface without causing damages (see Supplementary Information). This confirms that surface defects, as opposed to a uniform surface, are weak and more likely to be the initiation sites for the penetrating cracks that occur during lithium plating.

After the crack started to open and propagate in Stage II the increase in the force became moderate, and after $\sim 200 \mathrm{~s}$ the propagation slowed down while the crack continued to open. During this period, lithium started to plate along the crack wall perpendicular to the cantilever because of the compressive stress $(0.4-1.5 \mathrm{MPa}$ at the cantilever tip on $\mathrm{Li}),{ }^{39}$ preventing the increase in the stress from driving further propagation of the crack. In an actual lithium plating process, lithium will eventually fill the crack and cause the stress to rise again. To accelerate the process by increasing the compressive stress, the LPS particle was pushed against the cantilever by controlling the piezo manipulator of the holder. This is to simulate the increased concentration in the defect to evaluate the mechanical response of the crack propagation that leads to the penetration of lithium. During this rapid compression, there was no visible change in the geometries of the deposited lithium and the crack (see Supplementary Information for details). Note, during this compression, a positive bias was applied on the cantilever to prevent the lithium from plating.

After the additional compressive force was applied to the crack wall, the voltage was again set to negative to start lithium plating. Figure $3 \mathrm{~A}$ shows the evolution of the force and the change in the crack geometry during this second crack growth. (A combined plot for the initial crack growth, compression, and the second crack growth is included in the Supplementary Information.) The crack width continued to grow with a small increase in the crack depth. Lithium plating between the cantilever tip and the crack wall was visible as indicated by the arrowheads in Fig. 3B-D. The solid lines show that the tip of the cantilever was pushed away from the crack wall during the lithium plating. After 567s, the bias was reversed to strip the lithium, which resulted in the decrease of the force. However, both crack width and the length continued to increase. This implies the mechanical strength of the LPS was reduced after switching the bias to strip the plated lithium.

A Li concentration gradient within the solid electrolyte during charge/discharge was reported. ${ }^{49}$ This can cause a Li deficient layer in the LPS near the interface with Li, resulting in the weakening of the LPS. First 
principles simulations were performed to evaluate the effect of lithium concentration on the LPS structure and mechanical properties. LPS structures of differing concentrations of lithium atoms were subjected to tension. The original (stoichiometric) model (Fig. 4A) contained 24 lithium atoms and had a modulus of $26 \mathrm{GPa}$. The change in the lithium concentration caused a variation in the modulus from an $83 \%$ reduction $(1.9 \mathrm{GPa})$ to a $21 \%$ increase $(31 \mathrm{GPa})$ as shown in Fig. $4 \mathrm{~B}$, which plots the modulus at $1 \%$ strain versus the amount of lithium atoms present in the structure. This reduction in the modulus of the structures with significantly fewer lithium atoms suggests why crack growth was observed while the force on the crack was decreasing during lithium stripping.

Figure $4 \mathrm{C}$ compares the tensile stress-strain curves for the stoichiometric, lithium excess $(+4 \%)$ and lithium deficient (-4\%) LPS models. Adding and removing $1 \mathrm{Li}$ atom from the $24 \mathrm{Li}$-containing LPS model corresponds to $+4 \%$ and $-4 \%$ change in the Li concentration, respectively. The result shows that deviation (both addition and removal of $\mathrm{Li}$ ) from the stoichiometric structure severely weakens the strength of LPS. The changes in the atomic structures of the three models are shown in Fig. 4D. In the stoichiometric model the atomic bonds were uniformly stretched to the maximum stress at $35 \%$ strain. The breaking of the bonds took place equally across the cell as indicated by the dashed lines for $50 \%$ strain. Removing or adding just $4 \%$ of Li drastically imposes a disorder on the structure as seen in the $=0$ images in the figure. For the Li deficient structure (top panel), the dashed circle points to the location where one Li atom was removed. (The details of the structures seen from different orientations are shown in Supplementary Information.) At 25\% strain, where the maximum stress was attained, the atomic structure near the defect was clearly distorted in contrast to the stoichiometric case. After the stress drop was observed $(=40 \%)$, large voids were formed as the atoms repositioned from the dashed circles to the location indicated by the arrows. The Li excess structure (bottom panel) also exhibited distortion when an extra Li atom was added to the position indicated by the dashed line in red, and the distortion of the structure extended across the cell (see Supplementary Information for the structure viewed from different angles). As the strain increased and passed the maximum stress $(=20 \%)$, the atoms shifted from the green dashed circles to the positions indicated by the arrows at $30 \%$ strain, forming a large void. The added Li atom enhanced the local atomic bonds at the initial state leading to the increased modulus. However, the structural distortion imposed by the extra atom led to the non-uniform deformation and the formation of the void that decreased the tensile strength. The analysis of the change in the bond lengths clearly showed the non-uniform stretching and breaking of the atomic bonds, leading to local fracture that corresponds to the stress drops (see Supplementary Information for details).

These simulation results indicate that the mechanical stability of the off-stoichiometric LPS will decrease significantly regardless of whether it is Li deficient or in excess of Li. Therefore, high ionic conductivity is important to keep the LPS structure as neutral as possible during charge and discharge processes. The more that lithium atoms either "pile up" or "become depleted" in the LPS structure, the easier it is for the structure to fail. In addition, lithium deposition inside the LPS structure may lead to increased electronic conductivity, promoting lithium metal plating inside the LPS structure, ${ }^{50}$ causing a cascading avalanche effect of more lithium precipitation and penetrations accelerated by the reduced mechanical strength. 
This phenomenon may be more prevalent at the electrode interface regions, where lithium neutrality is most likely to deviate from the stoichiometric ratio due to the SSEs proximity to the lithium metal anode and lithium metal plated on the cathode. ${ }^{49}$ Massive depletion of Li causes low resistance against deformation. In fast charging or discharging situations, if an area of the SSE structure becomes lithium deficient, then the area may become weak, allowing for it to be a site for crack growth and trigger electrolyte fracture or Li penetrations.

\section{Conclusion}

In this work, an in situ TEM experiment was performed to directly observe and analyze the nucleation/propagation mechanisms of cracks in an SSE on the nano-scale, providing direct proof of the lithium penetration in SSEs due to nano-sized defects. During lithium plating, in areas of the LPS SSE where no major surface cracks were observed, lithium plated without visibly deforming the LPS. However, when lithium was deposited in a pre-existing defect at the LPS SSE surface, it was able to impart enough force on the surrounding LPS to enlarge the crack. Lithium grown in defect areas needed less force to initiate crack propagation than the maximum force observed by lithium plated at non-crack surfaces. It is worth noting that there was no external stress required for the lithium to push the crack open and propagate into the LPS.

The quantitative analysis of the force imposed on the crack wall during lithium plating revealed a threshold force for the crack to open. In addition, the crack kept growing even when the compressive force decreased during lithium stripping (where lithium ions are expected to migrate away from the Li/LPS interface), showing that lithium stripping can cause weakening of the LPS SSE. First principles simulations were used to evaluate the effect of lithium concentration on the mechanical property of LPS. It was shown that a lithium-deficient LPS exhibits a drastically reduced modulus, and the tensile strength is significantly decreased when the lithium concentration deviates from neutral stoichiometry. This suggests that weakening of LPS can occur when the stoichiometric ratio of lithium is not maintained, particularly when the lithium concentration is decreased.

The outcome of this study emphasizes the importance of minimizing the number of defects on the SSE surface, which can become initiation sites for crack growth and lithium penetration. It further suggests the necessity of maintaining the lithium concentration in the LPS SSE at its pristine state and preventing a large deviation in local lithium composition during cycling. Potential bottlenecks of lithium diffusion in the solid electrolyte, such as grain boundaries and heterointerfaces with the electrodes, can cause a nonequilibrium lithium concentration, further accelerating the degradation of the SSE and facilitating an increase in lithium penetrations.

This work also demonstrated the ability of in situ TEM to study the complex interplay between electrochemistry and mechanics and contribute towards solving some of the grand challenges in allsolid-state batteries. The technique introduced in this study paves a path toward a fundamental understanding of lithium penetrations in SSEs, and it has the potential for further expansion and 
development. For example, it can be combined with 3D tomography, nano-beam diffraction, or machinelearning approaches to clarify the 3-dimensional geometry and crystallographic orientations to provide a more detailed analysis of the phenomena. The quantitative analysis of the mechanical response of the SSE to the lithium plating and the potential change in the mechanical property during battery operation provide crucial information to simulate the charge/discharge processes and stability of ASSLBs.

\section{Declarations}

Acknowledgement

This work was supported by the National Science Foundation under Grant No. CMMI-1942554.

Contributions

M.A. and A.K. conceived the project and designed the experiments. M.A. and A.K. performed the in situ TEM experiments and ab initio simulations. Q.T. performed the fracture mechanics analysis. M.A. and A.K. wrote the paper. All authors discussed the results and commented on the paper.

\section{References}

1. Aurbach, D., Zinigrad, E., Cohen, Y. \& Teller, H. A short review of failure mechanisms of lithium metal and lithiated graphite anodes in liquid electrolyte solutions. Solid State lonics 148, 405-416 (2002).

2. Whittingham, M. S. History, Evolution, and Future Status of Energy Storage. Proceedings of the IEEE 100, 1518-1534 (2012).

3. Armand, M. \& Tarascon, J.-M. Building better batteries. Nature 451, 652-657 (2008).

4. Van Noorden, R. The rechargeable revolution: A better battery. Nature 507, 26-28 (2014).

5. Lithium Battery Incident Chart - FAA.

6. Could Chevy Volt Lithium-Ion Battery Fires Burn Out Interest in EVs and Hybrids? Scientific American (2011).

7. Tesla Says Car Fire Started in Battery. New York Times (2013).

8. Can you trust the lithium-ion battery in your pocket? The Washington Post (2016).

9. Lu, L., Han, X., Li, J., Hua, J. \& Ouyang, M. A review on the key issues for lithium-ion battery management in electric vehicles. Journal of Power Sources 226, 272-288 (2013).

10. Goodenough, J. B. \& Kim, Y. Challenges for Rechargeable Li Batteries. Chem. Mater. 22, 587-603 (2010). 
11. Fang, C., Wang, X. \& Meng, Y. S. Key Issues Hindering a Practical Lithium-Metal Anode. TRECHEM 1, 152-158 (2019).

12. Janek, J. \& Zeier, W. G. A solid future for battery development. Nature Energy 1, 16141 (2016).

13. Kerman, K., Luntz, A., Viswanathan, V., Chiang, Y.-M. \& Chen, Z. Review-Practical Challenges Hindering the Development of Solid State Li lon Batteries. J. Electrochem. Soc. 164, A1731-A1744 (2017).

14. Wenzel, S., Leichtweiss, T., Krüger, D., Sann, J. \& Janek, J. Interphase formation on lithium solid electrolytes - An in situ approach to study interfacial reactions by photoelectron spectroscopy. Solid State lonics 278, 98-105 (2015).

15. Wenzel, S. et al. Interphase formation and degradation of charge transfer kinetics between a lithium metal anode and highly crystalline Li7P3S11 solid electrolyte. Solid State Ionics 286, 24-33 (2016).

16. Zhu, Y., He, X. \& Mo, Y. First principles study on electrochemical and chemical stability of solid electrolyte-electrode interfaces in all-solid-state Li-ion batteries. J. Mater. Chem. A 4, 3253-3266 (2016).

17. Richards, W. D., Miara, L. J., Wang, Y., Kim, J. C. \& Ceder, G. Interface Stability in Solid-State Batteries. Chem. Mater. 28, 266-273 (2016).

18. Sudo, R. et al. Interface behavior between garnet-type lithium-conducting solid electrolyte and lithium metal. Solid State Ionics 262, 151-154 (2014).

19. Suzuki, Y. et al. Transparent cubic garnet-type solid electrolyte of Al2O3-doped Li7La3Zr2012. Solid State lonics 278, 172-176 (2015).

20. Sharafi, A., Meyer, H. M., Nanda, J., Wolfenstine, J. \& Sakamoto, J. Characterizing the LiLi7La3Zr2012 interface stability and kinetics as a function of temperature and current density. Journal of Power Sources 302, 135-139 (2016).

21. Aguesse, F. et al. Investigating the Dendritic Growth during Full Cell Cycling of Garnet Electrolyte in Direct Contact with Li Metal. ACS Appl. Mater. Interfaces 9, 3808-3816 (2017).

22. Basappa, R. H., Ito, T. \& Yamada, H. Contact between Garnet-Type Solid Electrolyte and Lithium Metal Anode: Influence on Charge Transfer Resistance and Short Circuit Prevention. J. Electrochem. Soc. 164, A666-A671 (2017).

23. Yubuchi, S. et al. Preparation of high lithium-ion conducting Li6PS5Cl solid electrolyte from ethanol solution for all-solid-state lithium batteries | Elsevier Enhanced Reader. Journal of Power Sources 293, 941-945 (2015). 
24. Yu, C., van Eijck, L., Ganapathy, S. \& Wagemaker, M. Synthesis, structure and electrochemical performance of the argyrodite Li6PS5Cl solid electrolyte for Li-ion solid state batteries. Electrochimica Acta 215, 93-99 (2016).

25. Boulineau, S., Courty, M., Tarascon, J.-M. \& Viallet, V. Mechanochemical synthesis of Li-argyrodite Li6PS5X $(X=\mathrm{Cl}, \mathrm{Br}, \mathrm{I})$ as sulfur-based solid electrolytes for all solid state batteries application. Solid State lonics 221, 1-5 (2012).

26. Bachman, J. C. et al. Inorganic Solid-State Electrolytes for Lithium Batteries: Mechanisms and Properties Governing Ion Conduction. Chemical Reviews 116, 140-162 (2016).

27. Sun, M. et al. Visualizing Lithium Dendrite Formation within Solid-State Electrolytes. ACS Energy Lett. 6, 451-458 (2021).

28. Wang, S. et al. High-Conductivity Argyrodite $\mathrm{Li}_{6} \mathrm{PS}_{5} \mathrm{Cl}$ Solid Electrolytes Prepared via Optimized Sintering Processes for All-Solid-State Lithium-Sulfur Batteries. ACS Appl. Mater. Interfaces 10, 4227942285 (2018).

29. Hänsel, C. \& Kundu, D. The Stack Pressure Dilemma in Sulfide Electrolyte Based Li Metal SolidState Batteries: A Case Study with Li6PS5Cl Solid Electrolyte. Advanced Materials Interfaces 8, 2100206 (2021).

30. McGrogan, F. P. et al. Compliant Yet Brittle Mechanical Behavior of Li2S-P2S5 Lithium-lonConducting Solid Electrolyte. Advanced Energy Materials 7, 1602011 (2017).

31. Tippens, J. et al. Visualizing Chemomechanical Degradation of a Solid-State Battery Electrolyte. ACS Energy Lett. 4, 1475-1483 (2019).

32. Han, F. et al. High electronic conductivity as the origin of lithium dendrite formation within solid electrolytes. Nature Energy 4, 187-196 (2019).

33. Liu, H. et al. Controlling Dendrite Growth in Solid-State Electrolytes. ACS Energy Lett. 5, 833-843 (2020).

34. Tian, H.-K., Xu, B. \& Qi, Y. Computational study of lithium nucleation tendency in Li7La3Zr2O12 (LLZO) and rational design of interlayer materials to prevent lithium dendrites. Journal of Power Sources 392, 79-86 (2018).

35. Liu, Q. et al. In Situ Observation of Sodium Dendrite Growth and Concurrent Mechanical Property Measurements Using an Environmental Transmission Electron Microscopy-Atomic Force Microscopy (ETEM-AFM) Platform. ACS Energy Lett. 5, 2546-2559 (2020).

36. LePage, W. S. et al. Lithium Mechanics: Roles of Strain Rate and Temperature and Implications for Lithium Metal Batteries. J. Electrochem. Soc. 166, A89-A97 (2019). 
37. Porz, L. et al. Mechanism of Lithium Metal Penetration through Inorganic Solid Electrolytes. Advanced Energy Materials 7, 1701003 (2017).

38. Sun, H. et al. In Situ Visualization of Lithium Penetration through Solid Electrolyte and Dead Lithium Dynamics in Solid-State Lithium Metal Batteries. ACS Nano acsnano.1c04864 (2021) doi:10.1021/acsnano.1c04864.

39. Diaz, M. \& Kushima, A. Direct Observation and Quantitative Analysis of Lithium Dendrite Growth by In Situ Transmission Electron Microscopy. J. Electrochem. Soc. 168, (2021).

40. He, Y. et al. Origin of lithium whisker formation and growth under stress. Nat. Nanotechnol. 14, 1042-1047 (2019).

41. Kresse, G. \& Hafner, J. Ab initio molecular dynamics for liquid metals. Phys. Rev. B 47, 558 (1993).

42. Kresse, G. \& Furthmüller, J. Efficient iterative schemes for ab initio total-energy calculations using a plane-wave basis set. Phys. Rev. B 54, 11169 (1996).

43. Perdew, J. P., Burke, K. \& Ernzerhof, M. Generalized Gradient Approximation Made Simple. Phys. Rev. Lett. 77, 3865 (1996).

44. Kresse, G. \& Joubert, D. From ultrasoft pseudopotentials to the projector augmented-wave method. Phys. Rev. B 59, 1758 (1999).

45. Blöchl, P. E. Projector augmented-wave method. Phys. Rev. B 50, 17953 (1994).

46. Monkhorst, H. J. \& Pack, J. D. Special points for Brillouin-zone integrations. Phys. Rev. B 13, 5188 (1976).

47. Ning, Z. et al. Visualizing plating-induced cracking in lithium-anode solid-electrolyte cells. Nat. Mater. 20, 1121-1129 (2021).

48. Garcia-Mendez, R., Smith, J. G., Neuefeind, J. C., Siegel, D. J. \& Sakamoto, J. Correlating Macro and Atomic Structure with Elastic Properties and Ionic Transport of Glassy Li2S-P2S5 (LPS) Solid Electrolyte for Solid-State Li Metal Batteries. Advanced Energy Materials 10, 2000335 (2020).

49. Santhanagopalan, D. et al. Interface Limited Lithium Transport in Solid-State Batteries. J. Phys. Chem. Lett. 5, 298-303 (2014).

50. Yue, J. \& Guo, Y.-G. The devil is in the electrons. Nat Energy 4, 174-175 (2019).

\section{Figures}



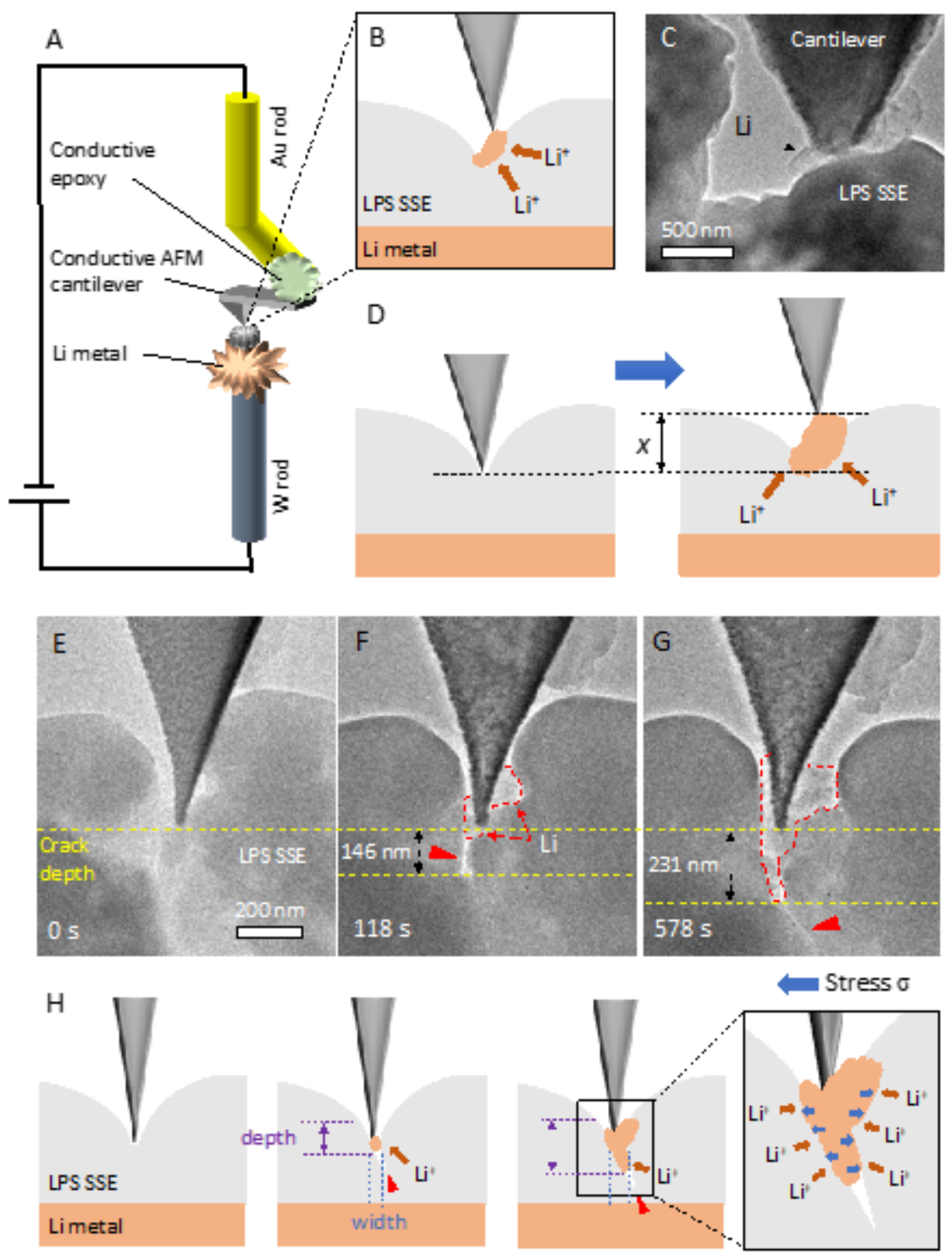

\section{Figure 1}

(A) Schematic illustration of the in situ TEM setup showing the AFM cantilever attached to a gold rod via a conductive epoxy approaching the SSE on lithium metal scratched onto a tungsten rod. (B) Magnified view of cantilever approaching the SSE. (C) TEM image of cantilever tip contacting LPS SSE showing lithium growth. (D) Schematic of cantilever displacement due to lithium dendrite growth upon biasing. (EG) TEM snapshots from video shown at different timestamps. Initially the cantilever and LPS SSE were aligned to obtain contact. At $118 \mathrm{~s}$ Li plating has occurred and the crack has started to open. At $578 \mathrm{~s} \mathrm{Li}$ plating has continued to widen the crack. Scale bar is $200 \mathrm{~nm}$. $(\mathrm{H})$ Schematic showing the crack increasing in both the depth and width directions, and the stress from the lithium pushing the crack apart as Li is deposited at the Li/SSE interface. 

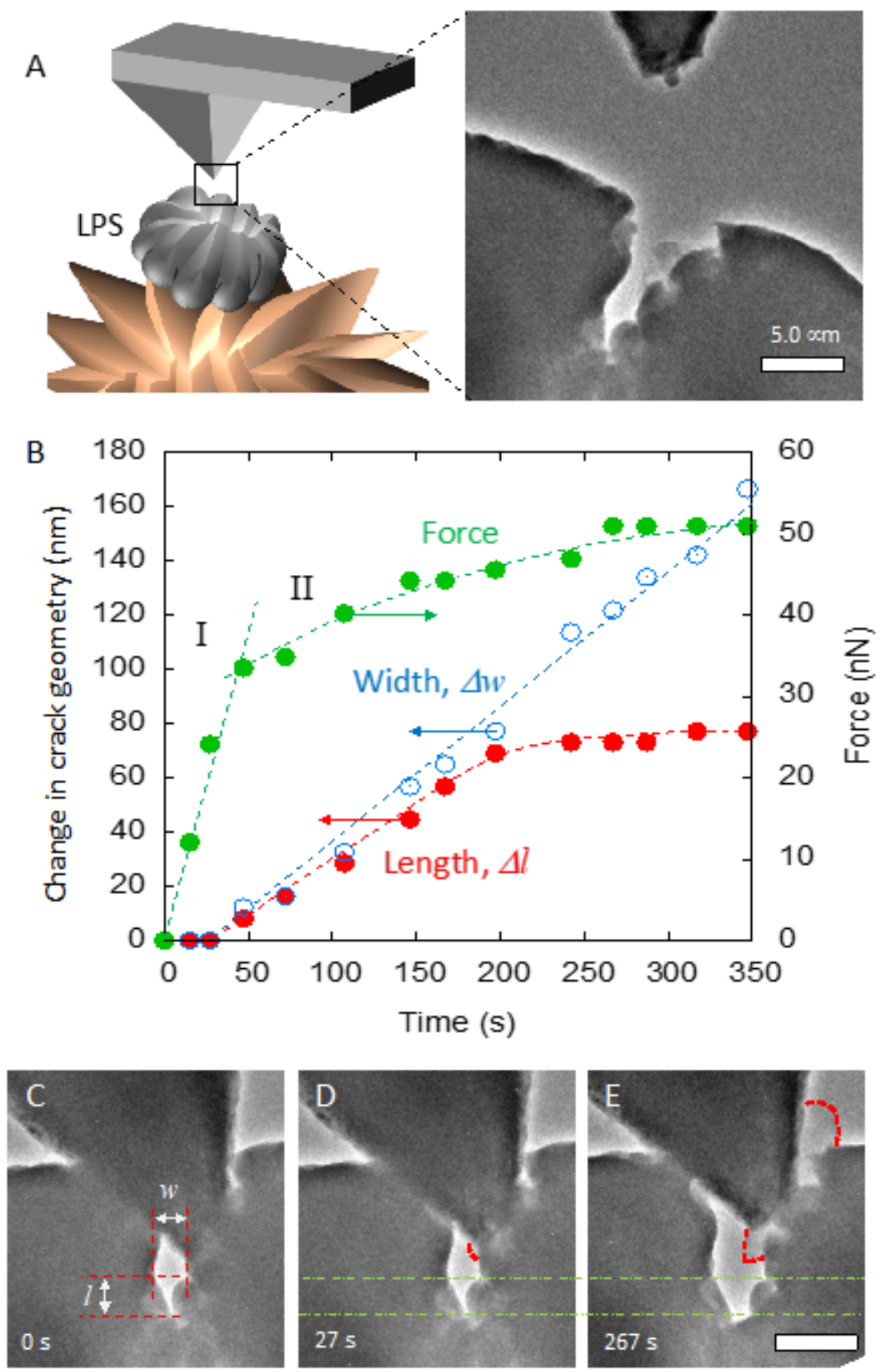

Figure 2

(A) Schematic illustration of conducting cantilever approaching a defect on LPS surface (left) and the TEM image (right). (B) Force evolution and change in the crack geometry during the initial lithium plating and crack growth. The force necessary for crack growth was $34 \mathrm{nN}$. (C) Initial alignment of the cantilever inside the crack. Dimensions of the crack width "w" and length "I" are labeled. (D) After 27s, the crack began to open. (E) Crack growth and lithium deposition is easily seen after 267s. Outline of the plated lithium is indicated by the dashed lines. The dash-dotted lines indicate the initial crack length, I, from B as a reference, and the scale bar is $500 \mathrm{~nm}$. 

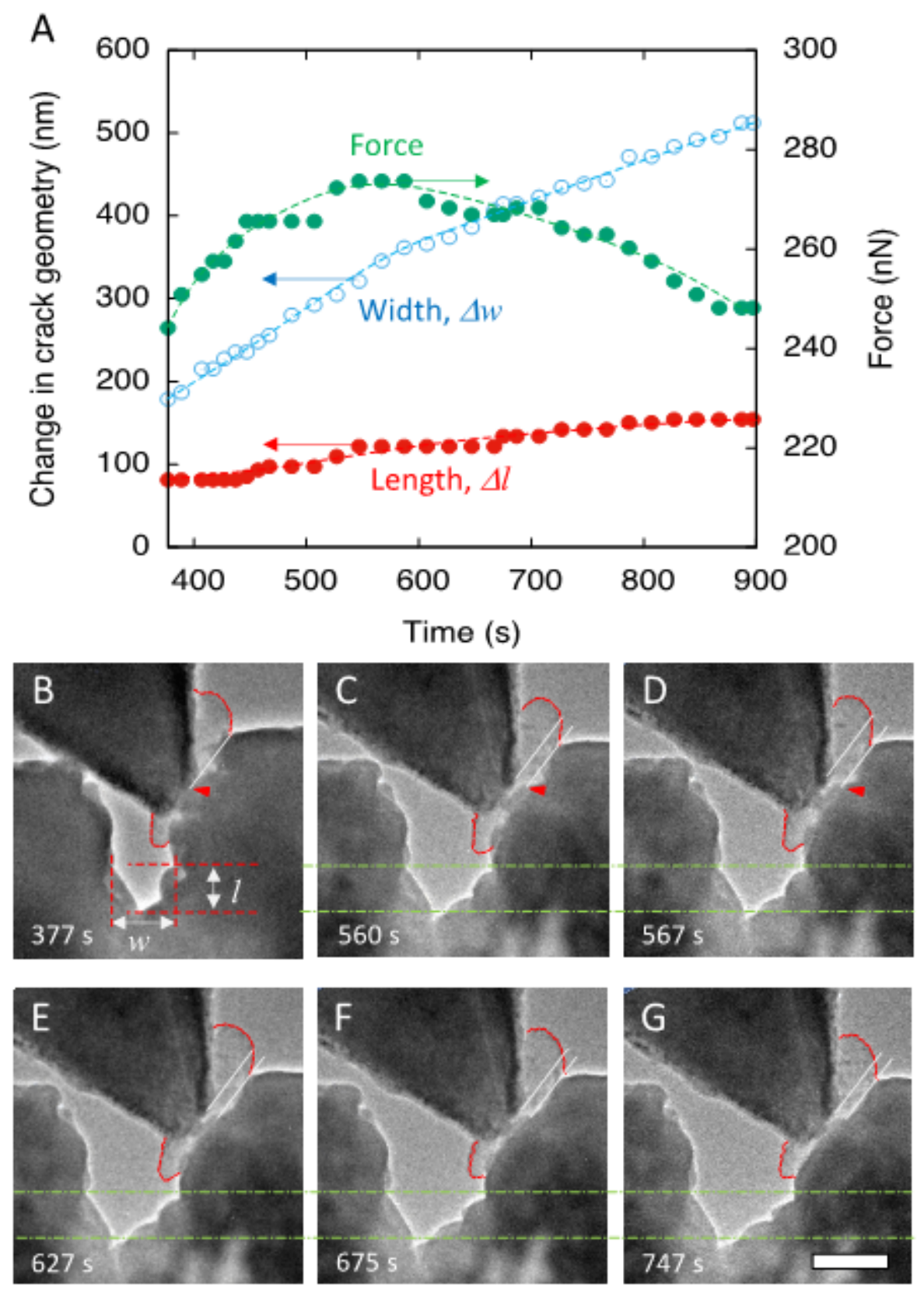

Figure 3

(A) Force evolution and change in the crack geometry during the second crack growth after additional compression was applied. (B-G) Snapshots of the crack opening process. Dimensions of the crack width " $\mathrm{W}$ " and length "I" are labeled in B. Outline of the plated lithium is indicated by the dashed lines. The dashdotted lines indicate the initial crack length, $l$, from $B$ as a reference, and the scale bar is $500 \mathrm{~nm}$. 


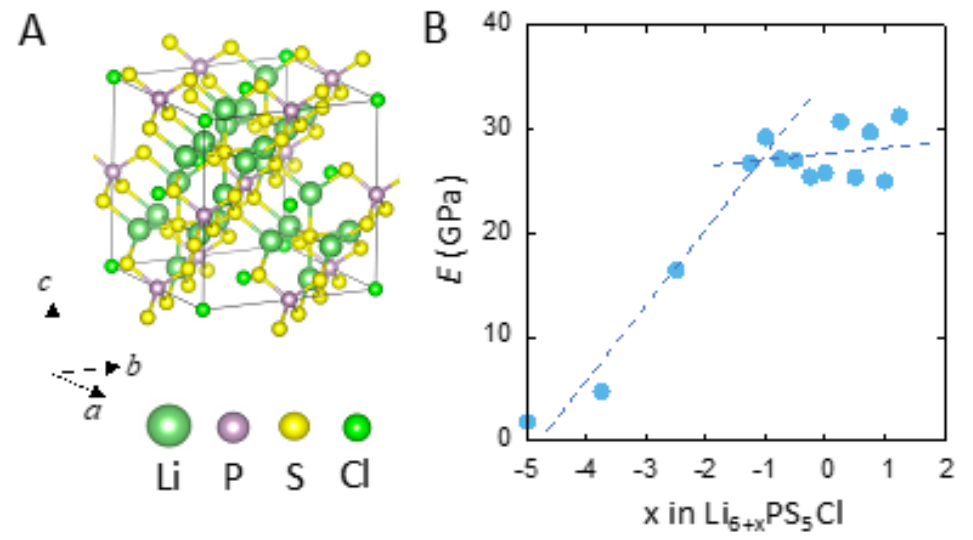

D
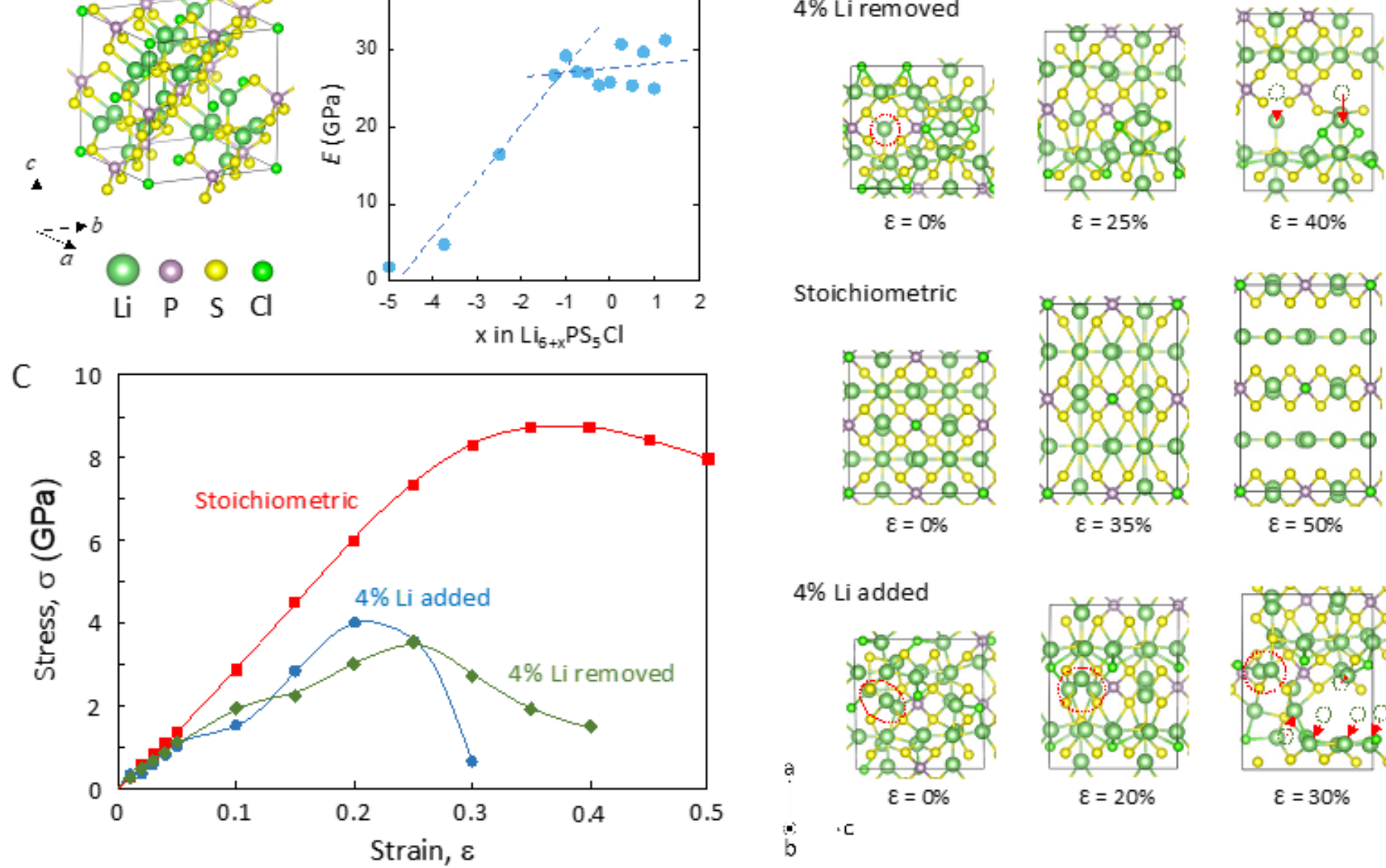

$\varepsilon=0 \%$

$\varepsilon=25 \%$

$\varepsilon=40 \%$

Stoichiometric
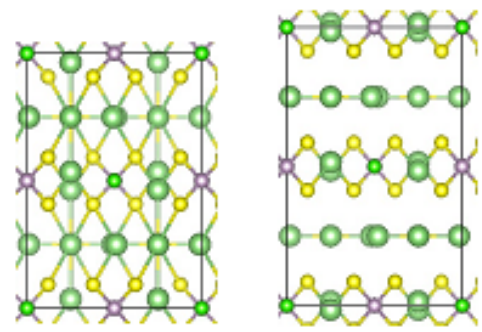

$\varepsilon=35 \%$

$\varepsilon=50 \%$

$4 \%$ Li added
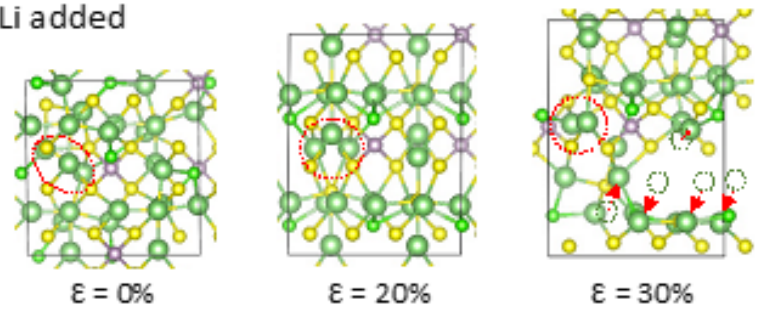

Figure 4

(A) Simulation mode of $\mathrm{Li}_{6} \mathrm{PS}_{5} \mathrm{Cl}$. (B) Modulus vs. Li concentration of LPS SSE. (C) The stress-strain curve of the LPS with stoichiometric and $\pm 4 \%$ Li concentrations. The yield stress drops significantly upon a $4 \%$ deviation from the stoichiometric lithium amount. (D) Structural changes of LPS during the tensile deformation at different Li concentrations.

\section{Supplementary Files}

This is a list of supplementary files associated with this preprint. Click to download.

- $S 12022.02 .09 . d o c x$

- MovieS1.mp4

- MovieS2.mp4

- MovieS3.mp4

- MovieS4.mp4

- MovieS5.mpg 
- MovieS6.mpg

Page 17/17 